\title{
Fortschritte, Probleme und zukünftige Aufgaben der Melissopalynologie
}

\author{
G Vorwohl \\ Universität Hohenheim, Landesanstalt für Bienenkunde, D-7000 Stuttgart 70, BRD
}

(Eingegangen 12 Juni 1990; angenommen 25 Juli 1990)

\begin{abstract}
Zusammenfassung - Seit der Veröffentlichung der ersten melissopalynologischen Arbeit durch Pfister (1895) sind nun fast 100 Jahre vergangen. Anna Maurizio hat einen beträchtlichen Teil ihrer wissenschaftlichen Arbeit der Entwicklung der Melissopalynologie gewidmet. Anlaß genug, die Fortschritte dieses Wissenszweiges, die Probleme der Honigherkunftsbestimmung und die zukünttigen Aufgaben der mikroskopischen Honiguntersuchung zu bedenken.
\end{abstract}

Melissopalynologie / Honig / botanische Herkunft / geographische Herkunft

\section{GEOGRAPHISCHE HERKUNFTSBESTIMMUNG}

Bei der Bestimmung der geographischen Herkunft der Honige gibt es keine grundsätzlichen Probleme. Das Pollenspektrum eines bestimmten Herkunftsgebietes ist weitgehend konstant. Dies zeigte sich bei der wiederholten Untersuchung der Honige des Trentino (Norditalien) durch Vorwohl (1972), Ricciardelli d'Albore (1981) und Lanzinger (1983). Es bestätigt sich auch immer wieder bei den Routineuntersuchungen im Labor.

Allerdings können Veränderungen in der landwirtschaftlichen oder forstlichen Nutzung zu langsamen, manchmal aber auch zu schubweisen Änderungen im Pollenbild der Honige führen. Die Ausweitung des Anbaus der Phacelia und die Einführung des Sonnenblumenanbaus in der Bundesrepublik hat binnen weniger
Jahre dazu geführt, daß die Pollen beider Pflanzen in Honigen der Bundesrepublik als Leitpollen auftreten können. Auch das gemeinsame Vorkommen von Phaceliaund Sonnenblumenpollen in Honigen aus der Bundesrepublik ist nichts ungewöhnliches mehr (Vorwohl, 1990). Vor wenigen Jahren wären solche Befunde als Verdachtsmomente für osteuropäische Herkunft gewertet worden.

Über die langfristigen Veränderungen im Pollenspektrum der Honige des Schweizer Mittellands haben Wille et al (1989) berichtet und Fossel (1974) über die Verschiebungen im Pollenmuster der Honige des steierischen Ennstals (Österreich). Neue Entwicklungen in der Land- und Forstwirtschaft müssen also im Auge behalten und Beurteilungsregeln gegebenenfalls revidiert werden.

Auch die Einführung neuer Zierpflanzen in Gärten, Friedhöfen und Parkanlagen solite verfolgt und der eventuelle Einflu $B$ 
auf das Pollenspektrum verifiziert werden. In Honigen aus dem ländlichen Raum verschwinden die Pollen der Zierpflanzen und Parkbäume in der Masse der Pollen der flächendeckenden Pflanzen. Anders liegen die Dinge bei den "Stadthonigen". Deren Pollenbild erweist sich aber als erstaunlich stabil (Maurizio, 1953; Vorwohl, 1980).

\section{ZUKÜNFTIGE AUFGABEN IM BEREICH DER GEOGRAPHISCHEN HERKUNFTSBESTIMMUNG}

Bei Anlegung strenger kritischer Maßstäbe wäre eine vollständige Übersicht über die Pollenspektren der Honige der Welt die Voraussetzung für die geographische Herkunftsbestimmung. Wenn heute ein Honig aufgrund seines Pollenmusters als aus dem tropisch-subtropischen Amerika stammend eingestuft wird, könnte der Einwand gemacht werden, wieso ausgeschlossen werden kann, daß er nicht $\mathrm{z} B$ aus Burma stammt. Dies ist $\mathrm{zwar}$ aller Erfahrung nach äußerst unwahrscheinlich, aber prinzipiell kann diese Kritik nicht von der Hand gewiesen werden, denn über die Honige Südostasiens haben wir nur sehr bescheidene Kenntnisse. Es gehört also zu den künftigen Aufgaben der Melissopalynologen, die Kenntnisse über die Pollenmuster der Honige der Länder zu vertiefen, die zur Zeit am Welthonighandel nicht beteiligt sind. Dies ist auch deshalb notwendig, weil in vielen Ländern der Dritten Welt imkerliche Entwicklungsprojekte laufen, die dazu führen könnten, daß Länder, die früher nur den Eigenbedarf deckten, zu Exportländern werden.

Neben der Ausweitung der Kenntnisse über die mikroskopischen Charakteristika der Honige der Welt ist eine Verfeinerung bei der Lokalisierung anzustreben. Unzureichend ausgearbeitet auf jeden Fall aber unzureichend publiziert sind die Unterscheidungsmöglichkeiten von benachbarten Regionen, die naturräumlich ähnlich aber durch politische Grenzen getrennt sind. Als Beispiele wären zu nennen: Die Unterscheidung von elsässischen (Frankreich) und badischen (Bundesrepublik Deutschland) Honigen oder die Abgrenzung von burgenländischen oder steirischen (Österreich) Honigen von denen der angrenzenden Nachbarländer. Prinzipiell erscheint das nicht aussichtslos. Die Laborerfahrung lehrt, daß z B zwischen hessischen und bayerischen (Bundesrepublik Deutschland) Honigen gewisse Unterschiede im Pollenmuster bestehen. In die gleiche Richtung laufen die Ergebnisse der Arbeit von Sturm (1988), der die Pollenspektren der oberösterreichischen Kalkalpen mit denen des Alpenvorlandes verglich. Diese Unterschiede müßten systematisch erfaßt werden. Dazu bedarf es der Untersuchung einer großen Zahl von authentischen Proben und der gründlichen Aufarbeitung der gewonnenen Pollenspektren. Die Komputertechnik gibt die Möglichkeit an die Hand, die anfallende Datenflut besser in den Griff zu bekommen.

\section{FORTSCHRITTE BEI DER GEOGRAPHISCHEN HERKUNFTSBESTIMMUNG}

Inzwischen liegen erste Arbeiten vor, die sich der Komputertechnik bedienen (Battesti, 1990; Perez de Zabalza, 1989; Spitzl, 1990). Überblickt man die Veröffentlichungen der letzten Jahre, kann man sagen, daß das Interesse an melissopalynologischen Arbeiten auch außerhalb der Bieneninstitute und 
lebensmittelchemischen Labors, die sich routinemäßig damit zu befassen haben, zugenommen hat. Als Beispiel sei die wachsende Zahl einschlägiger Publikationen in Spanien angeführt (Tabelle I).

Das Buch von Barth (1989) über die Pollen in Honigen Brasiliens zeigt, daß unsere Kenntnisse über die Pollenspektren der Honige der Welt auch im Bereich der Länder der dritten Welt und der Schwellenländer wachsen.

\section{PROBLEME DER BOTANISCHEN HERKUNFTSBESTIMMUNG}

Konnte eingangs gesagt werden, da $\beta$ bei der geographischen Herkunftsbestimmung keine grundsätzlichen Probleme auftauchen, so gilt dies für die Bestimmung der botanischen Herkunft, also der Nachprüfung der Sortenreinheit, leider nicht.

Schon Vergeron (1964) hat darauf verwiesen, daß die Zählergebnisse sehr stark fehlerbehaftet sind, wenn man, wie damals üblich, nur 100 Pollenkörner

Tabelle I. Melissopalynologie Veröffentlichungen in Spanien (ohne populärwissenschaftliche Artikel); aus Perez de Zabalza, 1989.

\begin{tabular}{lc}
\hline \multicolumn{1}{c}{ Jahr } & $\begin{array}{c}\text { Anzahl der } \\
\text { Publikationen }\end{array}$ \\
\hline vor 1952 & 3 \\
$1952-1960$ & 0 \\
$1961-1964$ & 2 \\
$1965-1968$ & 0 \\
$1969-1972$ & 1 \\
$1973-1976$ & 2 \\
$1977-1980$ & 4 \\
$1981-1984$ & 8 \\
seit 1984 & 25 \\
\hline
\end{tabular}

auszählt. Die Institute und Laboratorien, die sich in der Bundesrepublik mit Honiguntersuchungen befassen, haben Pollenzählungen im Ringversuch durchgeführt. Die Ergebnisse dürtten 1991 publikationsreif sein.

Die Zählgenauigkeit ist ein technisches Problem, das durch größeren Zählaufwand eingegrenzt werden kann. Es gibt aber bei der botanischen Herkunftsbestimmung Schwierigkeiten grundlegender Natur, die sich mit größerem Zählaufwand und mit verbesserter statistischer Absicherung der Zählergebnisse nicht beheben lassen.

Die botanische Herkunftsbestimmung beruht auf den markierenden Pollenkörnern, die von den Bienen zusammen mit dem Nektar eingetragen werden (primäre Einstäubung). Die Anzahl der Pollenkörner, die aus den Staubgefäßen in den Nektar gerät, hängt von der Anatomie der Blüte ab. Bekannte Extreme sind Myosotis, bei dem der Nektar hochgradig mit Pollen kontaminiert ist und Asphodelus, bei dem kaum Pollen in den Nektar gerät (Battesti, 1990).

Je nach der Größe der Pollenkörner und je nach ihrer Oberflächenbeschaffenheit greift der Schlundkopfmechanismus in der Honigblase unterschiedlich schnell. Als Regel kann gelten: je größer das Pollenkorn und je rauher seine Oberfläche, desto schneller die Eliminierung. Bestachelte Pollenkörner, wie die von Aster, Sonnenblume oder Löwenzahn, werden rascher aus dem Trachtgut entfernt als Pollenkörner gleicher Größe mit mehr oder minder glatter Oberfläche (Juntawong, 1989).

Aus dem Zusammenwirken der beiden Faktoren Blütenanatomie und Polleneliminierung im Proventriculus ergeben sich die Phänomene der Überund der Unterrepräsentierung des markierenden Pollen im Honig. 
Fast jeder Honig enthält Pollenköner nektarloser Pflanzen. Sie gelangen zum Teil als Luftsediment in den Honig. Insbesondere an Honigtau bleiben Pollenkörner haften, die in der Luft flottieren. Kommen die Pollenkörner nektarloser Pflanzen in höheren Prozentsätzen vor und stammen sie von Pollenspendern, die von den Bienen gerne genutzt werden, wie z B Filipendula, Plantago, Papaver, Fraxinus ornus, ist es unwahrscheinlich, daß es sich um "primäre" Einstäubung aus der Luft handelt. Louveaux hat schon 1958 gezeigt, daß im Bienenstock eine beachtliche sekundäre Einstäubung erfolgt mit Pollen, der von den Bienen getrennt von der Zuckerquelle direkt zur Eiweißversorgung eingetragen wird. Eine größere Zahl von Arbeiten zum Problem der sekundären Einstäubung wäre wünschenswert.

Daß beim Pressen des Honigs eine massive tertiäre Einstäubung aus zerdrücktem "Bienenbrot" stattfinden kann, weiß man schon lange. Dies ergibt sich schon aus dem Vergleich der Sedimentmengen von Press- und Schleuderhonigen und beim Vergleich der Pollendichten in den mikroskopischen Präparaten von Honigen der beiden Gewinnungsarten.

Tertiäre Einstäubung erfolgt aber nicht nur bei der Honiggewinnung durch Pressen.

Schwierigkeiten bei der Begutachtung französischer Heidehonige (von Calluna vulgaris, Besenheide) machten darauf aufmerksam, daß auch das bei dieser Honigsorte vor der Schleuderung notwendige "Stippen" zu einer tertiären Pollenanreicherung führt, weshalb nach anderen Parametern (Viskosität, Proteingehalt) für die Sortenabsicherung Ausschau gehalten wurde (Lunder, 1955; Louveaux, 1966).
Es hat den Anscinein, daß auch normale Schleuderhonige unterschiedlich stark tertiär eingestäubt sein können, und daß man nicht generell davon ausgehen kann, daß das Ausmaß der tertiären Kontamination immer in einer Größenordnung liegt, die vernachlässigt werden kann.

Bestimmte Honigherkünfte fallen durch hohen absoluten Pollengehalt auf, z B die spanischen Honigtauhonige aus Eichentracht. Diese Honige müßten als Honigtauhonige eher pollenarm sein. Man kann vermuten, daß die hohe absolute und relative Pollenzahl bei dieser Herkunft mit der weiten Verbreitung der Layens-Beute im Erzeugunsgebiet zusammenhängt, einer einräumigen Trogbeute ohne Trennung in Brut- und Honigraum. Ein beträchtlicher Teil der Honigwaben, die geschleudert werden, war daher vorher Brutwabe. Es ist daher wahrscheinlich, daß sie noch mehr eingelagerten Pollen enthalten als es bei Honigwaben aus dem Honigraum oder unbebrüteten Honigwaben üblich ist und daher die sekundäre und tertiäre Einstäubung begünstigen.

Die sekundäre und tertiäre Einstäubung mit Pollen nektarloser Pflanzen macht bei der Interpretation der botanischen Herkunft keine Schwierigkeiten. Ohne Zweifel beschränkt sich aber die sekundäre und tertiäre Einstäubung nicht auf die Pollen der nektarlosen Pflanzen, da viele Nektarspender gleichzeitig auch gute Pollenspender sind. Unter dem Mikroskop läßt sich nur das Vorhandensein eines Pollens konstatieren, aber nicht der Weg (primär, sekundär oder tertiär), auf dem er in den Honig gelangte. Die sekundäre und tertiäre Einstäubung kann daher zu Verzerrungen im Pollenspektrum führen, muß dies aber nicht zwangsläufig tun. Tragen die Bienen $\mathbf{z} B$ überwiegend Nektar von Weißklee ein und dient der Weißklee gleichzeitig als wichtigster 
Pollenspender, erfolgt die primäre Einstäubung mehrheitlich vom Weißklee. Auch auf sekundärem und später auf tertiärem Wege dürfte überwiegend Weißklee in den Honig gelangen. Die sekundäre und tertiäre Kontamination mit Pollen erhöht die absolute Anzahl der Pollen pro Gewichtseinheit Honig, sie ändert aber nichts an der Dominanz des Weißkleepollens im mikroskopischen Spektrum des Honigs. Die Interpretation des Pollenbildes nach den klassischen Regeln der Melissopalynologie liefert in diesem Fall das den Tatsachen entsprechende richtige Resultat.

Die sekundäre und tertiäre Einstäubung kann aber auch "gegenläufig" erfolgen. Ein bekanntes Beispiel sind die vorhin erwähnten Heidehonige aus den Landes bei Bordeaux. Die primär markierenden Calluna-Pollen werden prozentual durch Edelkastanienpollen zurückgedrängt, die aus den noch vorhandenen Bienenbrotvorräten aus der Edelkastanientracht auf sekundärem und vor allem auch auf tertiärem Wege durch das bei Heidehonig notwendige Stippen in den Honig gelangen. Dabei wird die Edelkastanie meist zum Leitpollen, der den markierenden Callunapollen "maskiert". Die Konsequenz aus diesem Sachverhalt ist die stärkere Heranziehung der chemischen, physikalischen, und sensorischen Eigenschaften zur Sortencharakterisierung.

Die mikroskopische Untersuchung wird dadurch nicht wertlos. Die Heidehonige der Landes bei Bordeaux haben durchaus ein typisches Pollenspektrum wie die Lüneburger Heidehonige oder die Honige anderer Heideregionen, das zur Sortencharakterisierung gehört.

Bei Honigtauhonigen liegen die Dinge etwas anders. Sekundäre und tertiäre Einstäubung verschieben zwar das Zahlenverhältnis zwischen Pollen nektarliefernder Pflanzen und Honigtauelementen (Pilze, Algen); was aber nicht verändert wird, ist die absolute Zahl der Honigtauelemente pro Gewichtseinheit Honig und die damit zusammenhängende Anzahl der Honigtauelemente pro mikroskopisches Blickfeld. Das Zahlenverhältnis Pollen nektarliefernder Pflanzen zu Honigtauanzeigern ist also mit Vorsicht zu interpretieren. Der Aussagewert der absoluten Zahl der Honigtauelemente pro Gewichtseinheit wird jedoch durch eventuelle sekundäre und tertiäre Einstäubung nicht beeinträchtigt, weil die Bienen Pilzelemente nicht höseln, womit diese von nicht-primären Kontaminationen ausgeschlossen sind. Eine Ausnahme machen die Rostsporen, die gelegentlich gesammelt werden. Sie lassen sich aber leicht als solche erkennen und gegebenenfalls bei der Zählung eliminieren.

\section{ZUKÜNFTIGE AUFGABEN AUF DEM GEBIET DER BOTANISCHEN HER- KUNFTSBESTIMMUNG}

$\mathrm{Zu}$ den vordringlichen Aufgaben gehört die Klärung der Auswirkung von Betriebsweise und Beutentypen auf das Ausmaß der tertiären Einstäubung.

Auch die Art der Entdeckelung (Gabel, Messer; Entdecklungsmaschine) scheint einen Einfluß auf die Pollenmenge zu haben, die auf tertiärem Wege in den Honig gelangt. Man hat den Eindruck, daß die maschinelle Entdeckelung zu verstärkter tertiärer Einstäuhung führt. Es fehlt dazu aber noch jede Dokumentation.

Da die generalisierende Anwendung einheitlicher Interpretationsregeln unter bestimmten Umständen (Maskierung der primären Einstäubung) in die Irre führen kann, sollte in Zukunft mehr Wert auf die Ausarbeitung regionaler Sortenspektren 
(z B französische Lavendelhonige aus der Provence) gelegt werden und zwar an Hand von authentischem Material, das durch sorgfältige Feldbeobachtungen abgesichert ist.

\section{Summary - Progress, problems and} future tasks of melissopalynology. Melissopalynology will soon be 100 years old. As far as geographical origin is concerned there are no basic problems. The pollen pattern of a certain region is quite stable. Changes in agriculture, however, like the introduction of Phacelia and sunflower into Western Germany lead to higher frequencies of both species in German honeys. The combination Phacelia/sunflower was considered in former years as typical for honeys from Eastern Europe.

We still have insufficient knowledge of the pollen spectra of the regions which do not participate in the world trade with honey, for instance Southeast Asia. Another open field of work is the differentiation between the honeys of adjacent domains eg, Southeastern Austria versus Hungary, Yugoslavia and Czechoslovakia. Computer techniques facilitate the handling of the many data accumulated on this kind of work (Perez de Zabalza, 1989; Battesti, 1990; Spitzl, 1990).

The determination of botanical origin is confronted with some fundamental problems. The countings are relatively inaccurate. The number of "marking" pollen grains in the nectar depends on the anatomy of the flowers. Size and ornamentation of the pollen grains influences the speed of elimination by the proventriculus mechanism in the honey bee stomach. There is a secondary contamination with pollen collected by the bees as protein food. A tertiary contamination may occur when the honey combs are uncapped and extracted. The grade of tertiary contamination seems to be influenced by the hive type, management and also by the uncapping technique used (fork, knife or uncapping machine).

Secondary and tertiary contamination can mask the marking pollen grains. Therefore, determination of botanical origin should also be considered together with the results of the sensory examination.

melissopalynology / honey / geographical origin / botanical origin

Résumé - Progrès, problèmes et futures tâches de la mélissopalynologie. La mélissopalynologie a bientôt 100 ans. Tant que l'on considère l'origine géographique, il n'y a pas de problèmes majeurs. Le spectre pollinique d'une région donnée est bien stable. Pourtant les changements en agriculture, tels que l'introduction de la phacélie ou du tournesol en Allemagne de l'Ouest, ont conduit à des fréquences plus élevées de ces 2 espèces dans les miels allemands.

Nous avons toujours une connaissance incomplète des spectres polliniques des régions qui ne participent pas au commerce international du miel, par exemple le Sud-Est asiatique. Un autre domaine ouvert aux recherches est la différenciation des miels provenant de régions voisines, par exemple le Sud-Est de l'Autriche et la Hongrie, la Yougoslavie et la Tchécoslovaquie. L'outil informatique facilite le traitement des données volumineuses accumulées dans ce genre de travail (Battesti, 1990; Perez de Zabalza, 1989; Spitlz, 1990).

La détermination de l'origine botanique se heurte à quelques problèmes fondamentaux. Les comptages sont relativement imprécis. Le nombre de grains de pollen "marqueur» dans le nectar dépend de l'anatomie des fleurs. La taille et l'orne- 
mentation des grains de pollen influencent leur vitesse d'élimination du proventricule dans l'estomac. II existe une contamination secondaire par le pollen récolté par les abeilles comme aliment protéiné. Une contamination tertiaire peut survenir quand les rayons sont désoperculés et le miel extrait. Le degré de contamination tertiaire semble être influencé par le type de ruche, les techniques de conduite et aussi la méthode utilisée pour le désoperculage (herse, couteau ou machine à désoperculer).

Les contaminations secondaire et tertiaire peuvent masquer les grains de pollen marqueur. La détermination de l'origine géographique ne devrait donc pas être basée uniquement sur l'examen microscopique, mais elle devrait prendre également en compte des paramètres chimiques et physiques ainsi que l'examen sensoriel.

\section{mélissopalynologie / miel / origine bota- nique / origine géographique}

\section{LITERATUR}

Es ist nicht die Absicht dieses Übersichtsartikels, eine vollständige Literaturliste zur Melissopalynologie zu liefern. Die zitierten Arbeiten liefern aber einen guten Einstieg in die weiterführende Lektüre.

Barth Monika (1989) O pólen no mel Brasileiro. Selbstverlag, Rio de Janeiro

Battesti MJ (1990) Contribution à la mélissopalynologie méditerranéenne. Les miels Corses. Thèse, Université d'Aix-Marseille III

Fossel A (1974) Die Bienenweide der Ostalpen, dargestellt am Beispiel des steirischen Ennstals. Mitt Naturwiss Ver Steiermak (Graz) 104, 87-118

Juntawong $N$ (1989) Verweildauer von Pollen bestimmter botanischer Herkunft in der Honigblase und im Honig bei Apis mellifera carnica L. Dissertation, Universität für Bodenkultur, Wien

Lanzinger MT (1983) Ricerca per la certificazione di origine e qualità dei mieli del Trentino. Tesi di Laurea, Milano

Louveaux J (1958) Recherches sur l'origine dans le miel du pollen de plantes entomophiles dépourvues de nectaires. Ann Abeille 2, 89-92

Louveaux J (1966) Essai de caractérisation des miels de Callune (Calluna vulgaris Salib). Ann Abeille 9 (4) 351-358

Lunder R (1953) Der Einfluß von Honiglösapparaten auf das Pollenbild des Heidehonigs. $Z$ Bienenforsch 3 (3), 49-52

Maurizio A (1953) Bäume und Sträucher als Bienenweide Schweiz Beitr Dendrol (5), 11-19

Perez de Zabalza A (1989) Estudio palinico de las mieles de Navarra. Tesis doctoral, Universidad de Navarra, Pamplona

Pfister R (1895) Versuch einer Mikroskopie des Honigs. Forschungsber Lebensmitt Bez Hyg Chem Pharm 2 (1), 25; 2 (2), 20

Ricciardelli d'Albore GC (1981) Nuove osservazioni microscopiche sui mieli del Trentino. Apic mod 72, 177-183

Spitzl K (1990) Das Pollenbild der fränkischen Honige. Apidologie, 21 (4) (im Druck)

Sturm J (1988) Beispiele zur Methodik der Honigpollenanalyse (Melissopalynologie) und die Pollenspektren einiger oberösterreichischer Honige. Diplomarbeit, Botanisches Institut der Universität für Bodenkultur, Wien

Vergeron P (1964) Interprétation statistique des résultats en matière d'analyses polliniques des miels. Ann Abeille 7 (4), 349-364

Vorwohl G (1972) Das Pollenbild der Honige aus den italienischen Alpen. Apidologie 2, 309-340

Vorwohl G (1980) Honig von der Bundesgartenschau. Biene 64, 167

Vorwohl G (1990) Veränderungen in Pollenbild der Honige der Bundesrepublik. Bienenvater 4, 143-147

Wille M, Wille $H$, Bogdanov S (1989) Welche Pollenarten enthalten die Honige aus dem Schweizerischen Mittelland. Vergleich der Honige aus den $30 e r$ und 80er Jahren. Schweiz Bienenztg 112 (3) 45-55; 112 (4) 215-225 